\title{
Longitudinal polarization analysis in small-angle neutron scattering
}

\author{
D. Honecker ${ }^{1}$, A. Ferdinand ${ }^{1}$, F. Döbrich ${ }^{1}$, C.D. Dewhurst ${ }^{2}$, A. Wiedenmann ${ }^{2}$, C. Gómez-Polo ${ }^{3}$, \\ K. Suzuki ${ }^{4}$, and A. Michels ${ }^{1, a}$ \\ 1 Technische Physik, Universität des Saarlandes, Postfach 151150, 66041 Saarbrücken, Germany \\ 2 Institut Laue-Langevin, 6 Rue Jules Horowitz, B.P. 156, 38042 Grenoble Cedex 9, France \\ 3 Departamento de Física, Universidad Pública de Navarra, Campus de Arrosadía, 31006 Pamplona, Spain \\ 4 Department of Materials Engineering, Monash University, Clayton, Victoria 3800, Australia
}

Received 4 March 2010 / Received in final form 19 April 2010

Published online 24 June 2010 - (C) EDP Sciences, Società Italiana di Fisica, Springer-Verlag 2010

\begin{abstract}
Due to recent progress in the development of ${ }^{3} \mathrm{He}$ spin filters, it has only now become possible to perform routinely longitudinal (one-dimensional) neutron-spin analysis (POLARIS) in small-angle neutron scattering (SANS) experiments. It is the purpose of this article to provide a brief introduction into the technique and to discuss first experimental data. In particular, for the most common scattering geometry where the applied magnetic (guide) field is perpendicular to the incident neutron beam, we write down the equations for the non-spin-flip and spin-flip SANS cross sections of a bulk ferromagnet, and we discuss the various angular anisotropies and asymmetries along with some selected experimental results on an $\mathrm{FeCr}$ based soft magnetic nanocrystalline alloy. In particular, we show that the analysis of the spin-flip data allows one to obtain the magnitude-squares of the three vector (Fourier) components of the magnetization.
\end{abstract}

\section{Introduction}

Polarized magnetic neutron scattering is one of the most powerful techniques for microstructure determination in magnetism research. The theoretical foundations have already been laid in 1939 in the seminal paper by Halpern and Johnson [1]. After the development of the first nuclear research reactors, the theory of polarized neutron scattering [2-7] has been worked out in the early 1960's and several classic experimental studies [8-15] have demonstrated the basic principles and paved the way for todays 3D cryogenic polarization-analysis device (CRYOPAD) $[12,16]$.

However, for the scattering of cold neutrons along the forward direction, it has only now become possible to perform routinely neutron-polarization analysis, for instance, at the SANS instrument D22 at the Institut Laue-Langevin, Grenoble, France [17]. This progress is mainly due to the recent development of efficient ${ }^{3} \mathrm{He}$ spin filters [18]. Consequently, only a few experimental SANS studies exist which do exploit this rather new option $[19,20]$. In this paper, we provide a brief introduction into the POLARIS technique, as it is applicable to SANS. The non-spin-flip and spin-flip cross sections (and linear combinations thereof) are expressed in a form which is

\footnotetext{
a Present address: Physics of Condensed Matter and Advanced Materials, University of Luxembourg, 162A Avenue de la Faïencerie, L-1511 Luxembourg, Luxembourg; e-mail: andreas.michels@uni.lu
}

adapted to the most commonly utilized SANS geometry, and selected experimental results on an FeCr based two phase nanocomposite alloy will be discussed. We would like to emphasize that we are not performing a $3 \mathrm{D}$ polarization analysis, but rather (in analogy to the experiment by Moon, Riste, and Koehler [9]) we measure scattering intensities which connect two neutron-spin states.

\section{Experimental setup}

The neutron experiments were perfomed at the SANS instrument D22 at the Institut Laue-Langevin [17]. The incident neutrons are monochromatized by means of a velocity selector. We used neutrons with a mean wavelength of $\lambda=8 \AA$ and with a wavelength spread of $\Delta \lambda / \lambda=10 \%$ (FWHM). A $1.2 \mathrm{~m}$ long remanent FeSi supermirror transmission polarizer $(m=3.6)$, installed immediately after the velocity selector, was used to select one of the two neutron-spin states. Reversal of the incident neutron polarization was achieved by a rf spin flipper which was installed close to the sample position. Measurement of the four partial neutron intensities $I^{++}, I^{--}, I^{+-}$, and $I^{-+}$(see below) became possible through a polarized ${ }^{3} \mathrm{He}$ spin-filter cell, which was placed inside a homogeneousfield cavity ("magic box"). Due to the rather large size of the magic box and in order to avoid depolarization of the cell due to stray magnetic fields, the analyzer device was 
installed in the evacuated detector tank, which in turn limited the accessible range of solid angles (minimum sampleto-detector distance $\sim 4 \mathrm{~m}$ ). The initial nuclear polarization of the ${ }^{3} \mathrm{He}$ spin filter is typically about $70 \%$, and the relaxation time of the ${ }^{3} \mathrm{He}$ nuclear magnetic moments is roughly 200-300 h [18]. Installation of the pre-polarized ${ }^{3} \mathrm{He}$ cell into the detector tank typically takes about $4 \mathrm{~h}$. Magnetic guide fields of the order of $1 \mathrm{mT}$ serve to maintain the polarization between polarizer, rf flipper, and ${ }^{3} \mathrm{He}$ filter. The scattered neutrons are detected by a multi-tube detector which consists of $128 \times 128$ pixels with a resolution of $8 \times 8 \mathrm{~mm}$. Raw data treatment was carried out by means of the GRASP [17] and BERSANS [21] software packages.

\section{POLARIS scattering cross sections for a bulk ferromagnet}

In the following, we aim at displaying the spin-resolved cross sections in a form which applies to the SANS geometry where the applied magnetic (guide) field $\mathbf{B}_{0} \| \mathbf{e}_{z}$ is normal to the wavevector $\mathbf{k}_{0}$ of the incident neutron beam. For $\mathbf{k}_{0} \| \mathbf{e}_{x}$, the scattering vector $\mathbf{q}$ (in the small-angle regime) varies in the $\mathbf{e}_{y}-\mathbf{e}_{z}$ plane, and the angle $\theta$ may be introduced in order to specify the orientation between $\mathbf{B}_{0}$ and q. For this geometry, the elastic differential POLARIS cross sections $d \Sigma / d \Omega$ can be written as $[9,15,22,23]$

$$
\begin{aligned}
\frac{d \Sigma^{ \pm \pm}}{d \Omega}(\mathbf{q})= & \frac{8 \pi^{3}}{V}\left(|\widetilde{N}|^{2}+b_{H}^{2}\left|\widetilde{M}_{z}\right|^{2} \sin ^{4} \theta\right. \\
& +b_{H}^{2}\left|\widetilde{M_{y}}\right|^{2} \sin ^{2} \theta \cos ^{2} \theta \\
& -b_{H}^{2}\left(\widetilde{M}_{y} \widetilde{M}_{z}^{*}+\widetilde{M}_{z} \widetilde{M}_{y}^{*}\right) \sin ^{3} \theta \cos \theta \\
& \mp b_{H}\left(\widetilde{N} \widetilde{M}_{z}^{*}+\widetilde{N}^{*} \widetilde{M}_{z}\right) \sin ^{2} \theta \\
& \left. \pm b_{H}\left(\widetilde{N} \widetilde{M}_{y}^{*}+\widetilde{N}^{*} \widetilde{M}_{y}\right) \sin \theta \cos \theta\right), \\
\frac{d \Sigma^{ \pm \mp}}{d \Omega}(\mathbf{q})= & \frac{8 \pi^{3}}{V} b_{H}^{2}\left(\left|\widetilde{M}_{x}\right|^{2}+\left|\widetilde{M}_{y}\right|^{2} \cos { }^{4} \theta\right. \\
& +\left|\widetilde{M}_{z}\right|^{2} \sin ^{2} \theta \cos ^{2} \theta \\
& \left.-\left(\widetilde{M}_{y} \widetilde{M}_{z}^{*}+\widetilde{M}_{z} \widetilde{M}_{y}^{*}\right) \sin \theta \cos ^{3} \theta\right) .
\end{aligned}
$$

Here, $V$ is the sample volume, $\widetilde{N}(\mathbf{q})$ and $\widetilde{\mathbf{M}}(\mathbf{q})=$ $\left[\widetilde{M}_{x}(\mathbf{q}), \widetilde{M}_{y}(\mathbf{q}), \widetilde{M}_{z}(\mathbf{q})\right]$ denote, respectively, the Fourier transforms of the nuclear scattering-length density and of the magnetization vector field $\mathbf{M}(\mathbf{x})=$ $\left[M_{x}(\mathbf{x}), M_{y}(\mathbf{x}), M_{z}(\mathbf{x})\right]$, and $b_{H}=2.7 \times 10^{-15} \mathrm{~m} / \mu_{B}$, where $\mu_{B}$ is the Bohr magneton. Helical scattering terms have been neglected in equation (2), and the magnetic form factor in the expression for the magnetic scattering amplitude was set to unity, which is permissible along the forward direction.

Note that the above POLARIS cross sections contain terms which are linear in the transversal Fourier coefficient $\widetilde{M}_{y}$, and that these may give rise to an asymmetric SANS pattern (compare Fig. 1 below). However, for polycrystalline ferromagnets with a random distribution

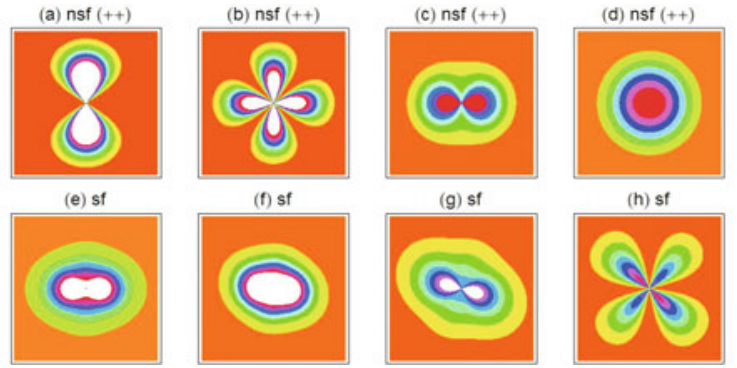

Fig. 1. (Color online) Qualitative variation of the neutron nonspin-flip (nsf) and spin-flip (sf) cross sections, equations (1) and (2), with the angle $\theta$ between the scattering vector $\mathbf{q}$ (inplane) and the applied magnetic field $\mathbf{B}_{0}$ (horizontal; pointing to the right). In these plots, we have neglected the possible $\theta$ dependence of the Fourier coefficients $\widetilde{N}(\mathbf{q})$ and $\widetilde{\mathbf{M}}(\mathbf{q})$, and we have explicitely taken into account the terms in the sf cross section that are linear in $\widetilde{M}_{y}$. Upper panel: nsf cross section $\frac{d \Sigma^{++}}{d \Omega}$ at magnetic saturation and for increasing ratio of $\widetilde{N} /\left(b_{H} \widetilde{M}_{z}\right)$ : (a) $\widetilde{N} /\left(b_{H} \widetilde{M}_{z}\right)=0.1$; (b) $\widetilde{N} /\left(b_{H} \widetilde{M}_{z}\right)=0.5$; (c) $\widetilde{N} /\left(b_{H} \widetilde{M}_{z}\right)=2.5$; (d) $\widetilde{N} /\left(b_{H} \widetilde{M}_{z}\right)=100$. Lower panel: qualitative field dependence of the sf cross section $\frac{d \Sigma^{+-}}{d \Omega}=\frac{d \Sigma^{-+}}{d \Omega} ; B_{0}$ increases from low fields (i.e., a few $\mathrm{mT}$ ) (e) to a few $10 \mathrm{mT}$ ((f) and (g)) to saturation (i.e., a few $10 \mathrm{~T}$ ) (h).

of perturbing magnetic anisotropy fields, the longitudinal magnetization component $M_{z}$ is positive (for sufficiently large positive applied fields) while $M_{y}$ takes on either sign with equal probability. Therefore, for isotropic polycrystals, terms such as $\widetilde{M}_{y} \widetilde{M}_{z} \sin ^{3} \theta \cos \theta$ and $\widetilde{N} \widetilde{M}_{y} \sin \theta \cos \theta$ may cancel and do generally not contribute to the scattering.

The four partial scattering cross sections are the two non-spin-flip (nsf) quantities, $\frac{d \Sigma^{ \pm \pm}}{d \Omega}$, and the two spin-flip (sf) cross sections $\frac{d \Sigma^{ \pm \mp}}{d \Omega}$. In particular, when the rf flipper is inactive (off), we measure, depending on the spin state of the ${ }^{3} \mathrm{He}$ filter, the nsf or sf intensity $\mathrm{I}^{++}$or $I^{+-}$, and when the flipper is active (on), we measure either $I^{--}$ or $I^{-+}$. However, since the transmission polarizer, the $\mathrm{rf}$ flipper, and the ${ }^{3} \mathrm{He}$ analyzer do not work perfectly, i.e., are characterized by efficiencies close to but smaller than unity, it is necessary to measure all four partial cross sections in order to correct for spin leakage [24]; such a correction can be done with the BERSANS software tool [21].

Due to the omission of chiral terms in equation (2), the two sf cross sections are independent of the polarization $P_{0}$ of the incident neutrons and expected to be identical (see below). On the other hand, the nsf channels contain two $P_{0}$ dependent terms. Furthermore, as a consequence of the trigonometric functions, a variety of angular anisotropies and asymmetries may be seen on a 2D detector (see below). Note that the information on the underlying magnetic microstructure is solely contained in the functions $\widetilde{\mathbf{M}}(\mathbf{q})$, which depend on the magnetic-interaction parameters (e.g., exchange, magnetostatics, magnetocrystalline and magnetoelastic anisotropy) and on the applied magnetic field. Therefore, by varying $B_{0}$ one can control the relative contributions of these terms to the POLARIS 
cross sections. For superparamagnetic particles, where the orientation distribution of the magnetic moments as a function of an effective magnetic field and temperature is determined by the Langevin statistics, the POLARIS cross sections have been derived and measured in references $[19,25]$. However, for a 3D bulk ferromagnet, the general POLARIS cross sections, equations (1) and (2), are determined by all three vector components of the Fourier coefficient of the magnetization.

The two SANSPOL cross sections $\frac{d \Sigma^{+}}{d \Omega}=\frac{d \Sigma^{++}}{d \Omega}+\frac{d \Sigma^{+-}}{d \Omega}$ and $\frac{d \Sigma^{-}}{d \Omega}=\frac{d \Sigma^{--}}{d \Omega}+\frac{d \Sigma^{-+}}{d \Omega}$ are easily recovered by adding the appropriate terms in equations (1) and (2). Furthermore, other useful relations are obtained by linear combinations of the partial cross sections [9], for instance,

$$
\begin{aligned}
& \frac{\frac{d \Sigma^{--}}{d \Omega}+\frac{d \Sigma^{++}}{d \Omega}}{2}= \frac{8 \pi^{3}}{V}\left(|\widetilde{N}|^{2}+b_{H}^{2}\left|\widetilde{M}_{z}\right|^{2} \sin ^{4} \theta\right. \\
&+b_{H}^{2}\left|\widetilde{M}_{y}\right|^{2} \sin ^{2} \theta \cos ^{2} \theta \\
&\left.-b_{H}^{2}\left(\widetilde{M}_{y} \widetilde{M}_{z}^{*}+\widetilde{M}_{z} \widetilde{M}_{y}^{*}\right) \sin ^{3} \theta \cos \theta\right), \\
& \frac{\frac{d \Sigma^{--}}{d \Omega}-\frac{d \Sigma^{++}}{d \Omega}}{2}= \frac{8 \pi^{3}}{V}\left(b_{H}\left(\widetilde{N} \widetilde{M}_{z}^{*}+\widetilde{N}^{*} \widetilde{M}_{z}\right) \sin ^{2} \theta\right. \\
&\left.-b_{H}\left(\widetilde{N} \widetilde{M}_{y}^{*}+\widetilde{N}^{*} \widetilde{M}_{y}\right) \sin \theta \cos \theta\right), \\
& \frac{\frac{d \Sigma^{--}}{d \Omega}+\frac{d \Sigma^{-+}}{d \Omega}+}{\frac{d \Sigma^{++}}{d \Omega}+\frac{d \Sigma^{+-}}{d \Omega}} 2 \frac{8 \pi^{3}}{V}\left(|\widetilde{N}|^{2}\right. \\
&+b_{H}^{2}\left|\widetilde{M}_{x}\right|^{2}+b_{H}^{2}\left|\widetilde{M}_{y}\right|^{2} \cos ^{2} \theta+b_{H}^{2}\left|\widetilde{M}_{z}\right|^{2} \sin { }^{2} \theta \\
&\left.\quad-b_{H}^{2}\left(\widetilde{M_{y}} \widetilde{M}_{z}^{*}+\widetilde{M}_{z} \widetilde{M}_{y}^{*}\right) \sin \theta \cos \theta\right) .
\end{aligned}
$$

The last expression denotes the total unpolarized scattering cross section $\frac{d \Sigma}{d \Omega}$. Equations (1)-(5) (in conjunction with a saturating magnetic field) permit the determination of "partial" correlation functions (see below).

Figure 1 depicts a few examples of the angular anisotropies and asymmetries of the nsf and sf SANS cross sections, which may be observed at different values of the applied magnetic field and momentum transfer. At complete magnetic saturation $\left(M_{x}=M_{y}=0\right)$, the magnetic SANS contrast originates from spatial variations $M_{s}(\mathbf{x})$ of the value of the saturation magnetization. In this particular case, the angular anisotropy of the nsf cross section $\frac{d \Sigma^{++}}{d \Omega}$ depends crucially on the relative magnitudes of nuclear to magnetic scattering: for small nuclear SANS $\left(\widetilde{N} /\left(b_{H} \widetilde{M}_{z}\right) \ll 1\right)$, one observes the well-known $\sin ^{4} \theta$ anisotropy of $\frac{d \Sigma^{++}}{d \Omega}$ (Fig. 1a). For increasing nuclear SANS, the terms $-b_{H} \widetilde{N} \widetilde{M}_{z} \sin ^{2} \theta$ in equation (1) become progressively important (Figs. 1b and 1c), and one finally observes isotropic nuclear scattering when $\widetilde{N} /\left(b_{H} \widetilde{M}_{z}\right) \gg 1$ (Fig. 1d). When the applied field is reduced, the transversal spin components give rise to the so-called spin-misalignment scattering in both the nsf and the sf channel, and an enhanced magnetic SANS signal may be observed along the field direction or along the diagonals of the detector. At saturation, the sf channel is expected to exhibit a $\sin ^{2} \theta \cos ^{2} \theta$ anisotropy (Fig. 1h). Note also that due to the terms which are uneven in the angle $\theta$, an asymmetric SANS pattern may be observed, in particular, when $\widetilde{M}_{y}$ and $\widetilde{M}_{z}$ are isotropic (e.g., Figs. if and $1 \mathrm{~g})$.

In the following, we will discuss a few experimental results which demonstrate the power of the SANS polarization-analysis technique for the investigation of magnetic microstructures.

\section{Some experimental results on an $\mathrm{FeCr}$ based nanocomposite}

The SANS measurements were carried out at room temperature and in the presence of a horizontal applied magnetic field. Given the limited beamtime and in an interest to explore a maximum range within the experimental parameter space $\left(q, B_{0}\right.$, neutron-spin state), we display in some instances raw data.

Figure 2 depicts SANS results which were obtained on a two-phase FeCr based nanocomposite alloy with an average particle size of $D=10-15 \mathrm{~nm}$. The volume fraction of the crystalline particle phase is about $30 \%$. For more details on sample characterization, we refer to reference [26].

At a saturating field of $1.31 \mathrm{~T}$ (Figs. $2 \mathrm{a}$ and $2 \mathrm{~b}$ ), both nsf cross sections $\frac{d \Sigma^{ \pm \pm}}{d \Omega}$ exhibit a strongly enhanced signal normal to the field direction, in qualitative agreement with the $\sin ^{4} \theta$ prediction by equation (1). Furthermore, the results in Figures $2 \mathrm{a}$ and $2 \mathrm{~b}$ clearly reveal that the nuclear SANS intensity of this alloy is very much smaller than the magnetic scattering (compare also Figs. 1a-1d); this statement was also verified by measuring the unpolarized cross section at saturation (cf. Eq. (5)). At lower fields, transversal spin components contribute to both the nsf and sf scattering, and in both nsf channels (Figs. 2c and $2 \mathrm{~d}$ ) one still observes a dominating scattering perpendicular to $\mathbf{B}_{0}$. Closer inspection indicates, however, that the pattern is now slightly asymmetric (tilted) with respect to the vertical in-plane direction. The asymmetry of the signal is a consequence of the spin-misalignment scattering, which enters equation (1) through terms that are uneven in the angle $\theta$. Since the nuclear SANS is much smaller than the magnetic scattering, the polarizationindependent terms $\widetilde{M}_{y} \widetilde{M}_{z} \sin ^{3} \theta \cos \theta$ in equation (1) are most likely responsible for the asymmetry. As already discussed above, such an asymmetry is not expected for an isotropic polycrystalline magnet, and its existence in the data is therefore indicative of the presence of texture in the magnetic microstructure. However, an unambiguous confirmation of this asymmetry is the subject of further investigations.

The sf cross section $\frac{d \Sigma^{+-}}{d \Omega}$ at saturation (Fig. 2e) shows the characteristic $\sin ^{2} \theta \cos ^{2} \theta$ variation with maxima along the diagonals of the detector $\left(\theta= \pm 45^{\circ}\right)$, while $\frac{d \Sigma^{+-}}{d \Omega}$ at $0.07 \mathrm{~T}$ (Fig. 2f) exhibits a dominating scattering parallel to $\mathbf{B}_{0}$. A decisive advantage of the present polarization-analysis technique compared to the usual unpolarized or polarized incident-beam experiment becomes obvious: measurement of the sf channel at 

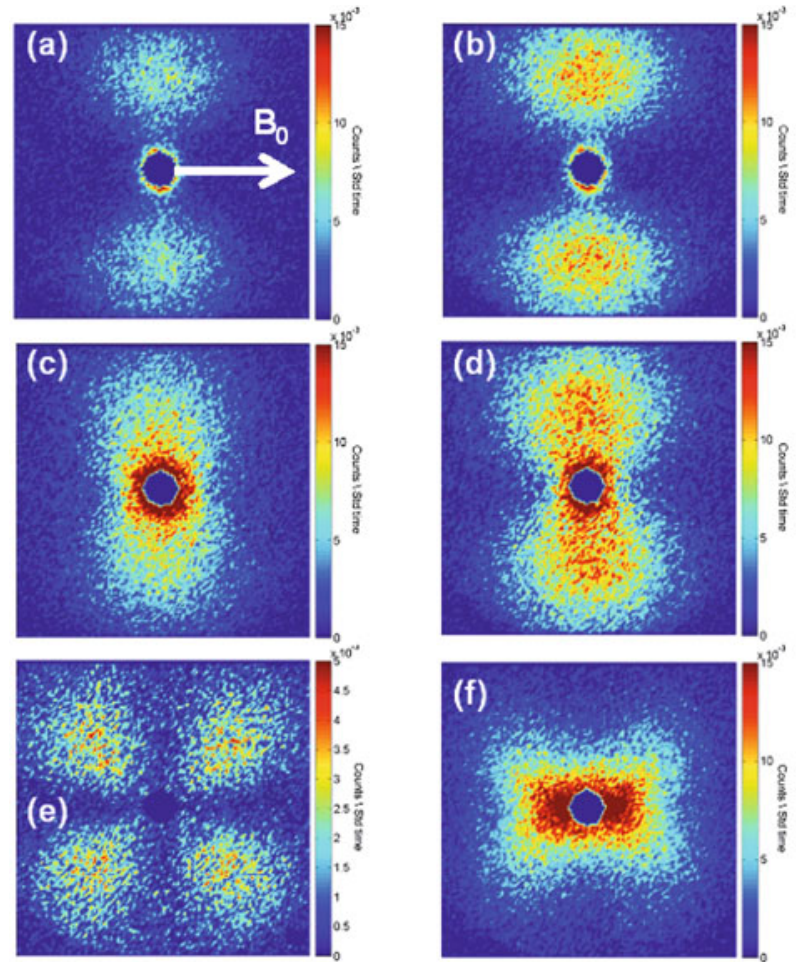

Fig. 2. (Color online) SANS polarization-analysis results on an $\mathrm{FeCr}$ based nanocomposite. The direction of the applied magnetic field $\mathbf{B}_{0}$ is horizontal (pointing to the right). Neutron non-spin-flip cross sections $\frac{d \Sigma^{++}}{d \Omega}$ (a) and $\frac{d \Sigma^{--}}{d \Omega}$ (b) at $B_{0}=$ $1.31 \mathrm{~T}$. (c) $\frac{d \Sigma^{++}}{d \Omega}$ and (d) $\frac{d \Sigma^{--}}{d \Omega}$ at $B_{0}=0.02 \mathrm{~T}$. $\frac{d \Sigma^{+-}}{d \Omega}$ at $B_{0}=$ $1.31 \mathrm{~T}(\mathrm{e})$ and at $B_{0}=0.07 \mathrm{~T}(\mathrm{f})$. The range of momentum transfers (from the center to the corner of the detector) roughly corresponds to $0.04 \mathrm{~nm}^{-1}<q<0.55 \mathrm{~nm}^{-1}$.

saturation yields the partial correlation function $\left|\widetilde{M}_{z}\right|^{2}$, without the nuclear scattering. Moreover, since the angular anisotropy of the pattern in Figure 2e is nearly exclusively of the $\sin ^{2} \theta \cos ^{2} \theta$ type, this strongly suggests that $\widetilde{M}_{z}$ is isotropic, i.e., $\widetilde{M}_{z}(\mathbf{q})=\widetilde{M}_{z}(q)$. This assessment is further supported by the results shown in Figure 3.

In Figure 4, we show the radially-averaged sf cross sections $\frac{d \Sigma^{+-}}{d \Omega}$ and $\frac{d \Sigma^{-+}}{d \Omega}$ at $1.31 \mathrm{~T}$. These data have been corrected for spin leakage and demonstrate that the scattering in both sf channels is equal, as is expected for this type of polycrystalline alloy.

Figure 5 displays the radially-averaged sf intensity at different applied magnetic fields. The sf signal is strongly field dependent, in qualitative agreement with previous results for the spin-misalignment scattering of nanocrystalline ferromagnets [22]. The data at the largest field exhibit a peak at $q_{p} \cong 0.22 \mathrm{~nm}^{-1}\left(2 \pi / q_{p}=30 \mathrm{~nm}\right)$, which may be attributed to dense packing and/or diffusion zones around the particles [27].

The spin-misalignment scattering can be further analyzed by calculating appropriate averages along certain directions in momentum space. Figure 6 shows radial sector averages of the $2 \mathrm{D}$ sf data along the vertical $\left(\theta=90^{\circ}\right)$ and the horizontal $\left(\theta=0^{\circ}\right)$ direction. By comparison to

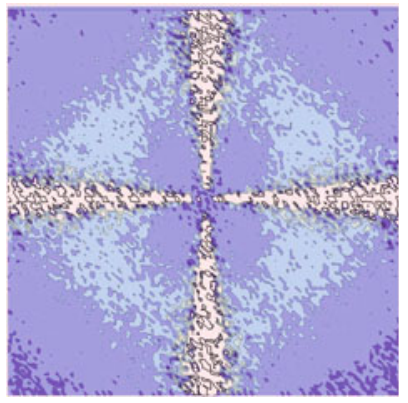

Fig. 3. (Color online) The function $\left|\widetilde{M}_{z}(\mathbf{q})\right|^{2}$ of an FeCr based nanocomposite at $B_{0}=1.31 \mathrm{~T}$. $\left|\widetilde{M}_{z}\right|^{2}$ was determined from the spin-flip cross section $\frac{d \Sigma^{+-}}{d \Omega}$. $q$-range is $0.04 \mathrm{~nm}^{-1}<q<$ $0.55 \mathrm{~nm}^{-1}$. The white horizontal $\left(\theta=0^{\circ}\right.$ and $\left.\theta=180^{\circ}\right)$ and vertical $\left(\theta=90^{\circ}\right.$ and $\left.\theta=270^{\circ}\right)$ lines are an artifact which results from the division of the corresponding anisotropic data by $\sin ^{2} \theta \cos ^{2} \theta$.

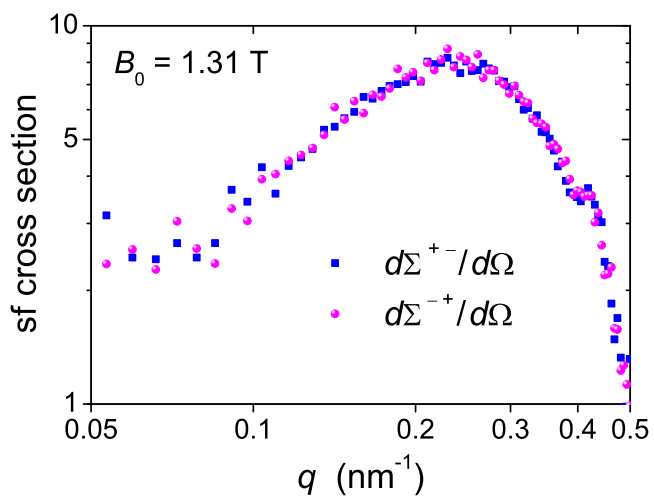

Fig. 4. (Color online) Radially-averaged spin-flip cross sections $\frac{d \Sigma^{+-}}{d \Omega}$ and $\frac{d \Sigma^{-+}}{d \Omega}$ of an FeCr based nanocomposite at $B_{0}=1.31 \mathrm{~T}$.

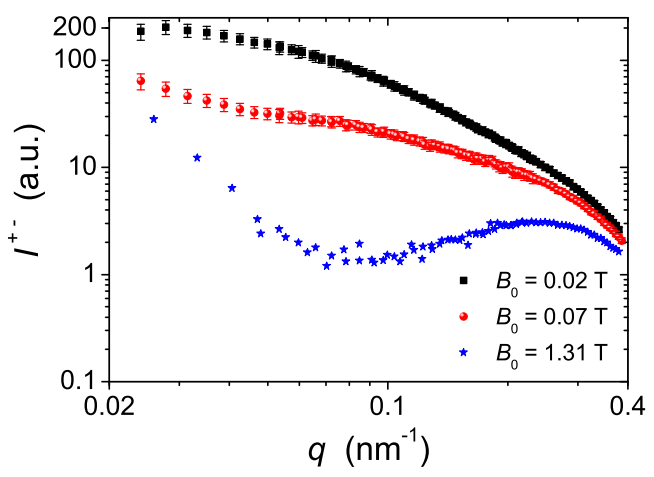

Fig. 5. (Color online) Radially-averaged spin-flip intensity $I^{+-}$of an FeCr based nanocomposite at different applied magnetic fields.

equation (2) it is seen that these averages are, respectively, approximations to the $\left|\widetilde{M}_{x}\right|^{2}$ and to the $\left|\widetilde{M}_{x}\right|^{2}+\left|\widetilde{M}_{y}\right|^{2}$ correlation functions. The transversal correlation functions vary by almost two orders of magnitude between the highest field of $1.31 \mathrm{~T}$ and $20 \mathrm{mT}$ (compare Fig. 4). Roughly, the $\left|\widetilde{M}_{x}\right|^{2}+\left|\widetilde{M}_{y}\right|^{2}$ data appear to be twice as large as $\left|\widetilde{M}_{x}\right|^{2}$. 


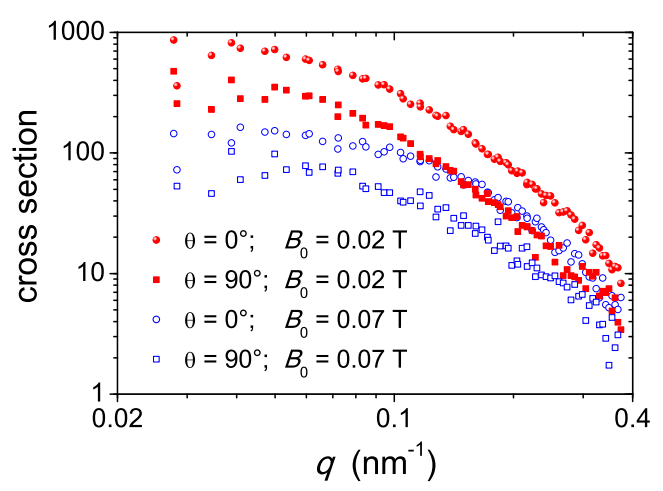

Fig. 6. (Color online) Field dependence of $\left( \pm 7.5^{\circ}\right)$ sector averages of the spin-flip cross section of an FeCr based nanocomposite. The curves labelled $\theta=90^{\circ}$ are related to $\left|\widetilde{M}_{x}\right|^{2}$, while the $\theta=0^{\circ}$ data is proportional to $\left|\widetilde{M}_{x}\right|^{2}+\left|\widetilde{M}_{y}\right|^{2}$ (compare Eq. (2)).

\section{Summary and conclusions}

We have provided a brief introduction into longitudinal neutron-spin analysis (POLARIS), as applicable to a small-angle neutron scattering (SANS) experiment. Such type of studies can now be carried out routinely at the SANS instrument D22 at the Institut Laue-Langevin. The general POLARIS cross sections for a bulk ferromagnet have been displayed in a form which is adapted to the most common SANS geometry where the applied magnetic field is normal to the incident neutron beam. By means of this method it becomes possible to measure partial correlation functions, and several novel anisotropies and asymmetries in the magnetic scattering cross section may be observed. For the example of an FeCr based two-phase nanocrystalline alloy, we have shown that the (isotropic) longitudinal correlation function $\left|\widetilde{M}_{z}\right|^{2}$ as well as the transversal contributions $\left|\widetilde{M}_{x}\right|^{2}$ and $\left|\widetilde{M}_{y}\right|^{2}$ can be extracted from the spin-flip cross section. Such a procedure opens up the unique possibility for analyzing independently the three vector components of the bulk magnetization, and we believe that this technique will significantly contribute to the understanding of the magnetic interactions in nanostructures.

We thank the Institut Laue-Langevin and the Deutsche Forschungsgemeinschaft (Grant No. MI 738/6-1) for financial support. Fruitful discussions with Joachim Kohlbrecher are gratefully acknowledged.

\section{References}

1. O. Halpern, M.H. Johnson, Phys. Rev. 55, 898 (1939)

2. A.W. Sáenz, Phys. Rev. 119, 1542 (1960)

3. S.V. Maleyev, Sov. Phys. JETP 13, 860 (1961)

4. Y.A. Izyumov, S.V. Maleyev, Sov. Phys. JETP 14, 1168 (1962)

5. S.V. Maleyev, V.G. Baryakhtar, R.A. Suris, Sov. Phys. Solid State 4, 2533 (1963)

6. M. Blume, Phys. Rev. 130, 1670 (1963)

7. W. Marshall, R.D. Lowde, Rep. Prog. Phys. 31, 705 (1968)

8. C.G. Shull, E.O. Wollan, W.C. Koehler, Phys. Rev. 84, $912(1951)$

9. R.M. Moon, T. Riste, W.C. Koehler, Phys. Rev. 181, 920 (1969)

10. M. Th. Rekveldt, J. Phys. 32, C1 (1971)

11. G.M. Drabkin, A.I. Okorokov, V.V. Runov, Sov. Phys. JETP Lett. 5, 324 (1972)

12. A.I. Okorokov, V.V. Runov, A.G. Gukasov, Nucl. Instr. Meth. 157, 487 (1978)

13. A.I. Okorokov, V.V. Runov, B.P. Toperverg, A.D. Tretyakov, E.I. Maltsev, I.M. Puzei, V.E. Mikhailova, JETP Lett. 43, 503 (1986)

14. F. Mezei, Physica B 137, 295 (1986)

15. O. Schärpf, H. Capellmann, Phys. Status Solidi A 135, 359 (1993)

16. F. Tasset, Physica B 156-157, 627 (1989)

17. For more details, see, http://www.ill.eu/d22.

18. A.K. Petoukhov, V. Guillard, K.H. Andersen, E. BourgeatLami, R. Chung, H. Humblot, D. Jullien, E. LelievreBerna, T. Soldner, F. Tasset, M. Thomas, Nucl. Instr. Meth. A 560, 480 (2006)

19. U. Keiderling, A. Wiedenmann, A. Rupp, J. Klenke, W. Heil, Meas. Sci. Technol. 19, 034009 (2008)

20. K.L. Krycka, R. Booth, J.A. Borchers, W.C. Chen, C. Conlon, T.R. Gentile, C. Hogg, Y. Ijiri, M. Laver, B.B. Maranville et al., Physica B 404, 2561 (2009)

21. U. Keiderling, Appl. Phys. A 74, S1455 (2002)

22. A. Michels, J. Weissmüller, Rep. Prog. Phys. 71, 066501 (2008)

23. J. Schweizer, in Neutron Scattering from Magnetic Materials, edited by T. Chatterji (Elsevier, Amsterdam, 2006), pp. $153-213$

24. A.R. Wildes, Neutron News 17, 17 (2006)

25. A. Wiedenmann, Physica B 356, 246 (2005)

26. C. Gómez-Polo, L.M. Socolovsky, J.C. Denardin, M. Knobel, J.I. Pérez-Landazábal, V. Recarte, J. Magn. Magn. Mater. 316, e876 (2007)

27. A. Heinemann, H. Hermann, A. Wiedenmann, N. Mattern, K. Wetzig, J. Appl. Cryst. 33, 1386 (2000) 